\title{
Differential Effects of Glucocorticoid and Mineralocorticoid Antagonism on Anxiety Behavior in Mild Traumatic Brain Injury
}

\author{
Laura C. Fox, Daniel R. Davies, Jamie L. Scholl, Michael J. Watt, Gina L. Forster* \\ Affiliations: \\ Center for Brain and Behavior Research, at the University of South Dakota Sanford \\ School of Medicine, Division of Basic Biomedical Sciences, 414 East Clark St, \\ Vermillion, SD, United States \\ Total number of Pages: 10 \\ Total number of Words (3500 word limit): 3045 \\ Number of Words in Abstract (250 word limit): 180 \\ Number of Tables or Figures (limit of 4): 1 Figure \\ Number of References (25 limit): 14 \\ ${ }^{*}$ Corresponding Author \\ Gina L. Forster, Ph.D. \\ Division of Basic Biomedical Sciences \\ Sanford School of Medicine at the University of South Dakota \\ 414 E. Clark St. \\ Vermillion, SD, 57069 \\ USA \\ Telephone: (605) 6776883 \\ Fax: (605) 6776381 \\ Email: gforster@usd.edu
}

Email Addresses of Other Authors: laura.fox@usd.edu,

Daniel.davies@coyotes.usd.edu, Jamie.Scholl@usd.edu, and Mick.Watt@usd.edu

(C) 2016. This manuscript version is made available under the Elsevier user license

http://www.elsevier.com/open-access/userlicense/1.0/ 


\section{Abstract}

Mild traumatic brain injuries (TBIs) comprise three-quarters of all TBls occurring in the United States annually, and psychological symptoms arising from them can last years after injury. One commonly observed symptom following mild TBI is generalized anxiety. Most mild TBIs happen in stressful situations (sports, war, domestic violence, etc.) when glucocorticoids are elevated in the brain at the time of impact, and glucocorticoids have negative effects on neuronal health following TBI. Therefore, blocking glucocorticoid receptors might prevent emergence of anxiety symptoms post-injury. Adult male rats received mifepristone $(20 \mathrm{mg} / \mathrm{kg})$ or spironolactone $(50 \mathrm{mg} / \mathrm{kg})$ to block glucocorticoid and mineralocorticoid receptors, respectively, $40 \mathrm{~min}$ prior to being exposed to acute social defeat stress followed immediately by mild TBI. In defeated rats with concomitant mild TBI, mifepristone restored time spent in the open arms of an elevated plus maze to control levels, demonstrating for the first time that glucocorticoid receptors play a critical role in the development of anxiety after mild TBI. Future treatments could target these receptors, alleviating anxiety as a major side effect in victims of mild TBI sustained in stressful situations.

Keywords: Glucocorticoids, mild traumatic brain injury, anxiety, stress, rat 
Mild traumatic brain injuries (TBIs) are common occurrences in sports, warfare and everyday life, and comprise $75-80 \%$ of total TBIs sustained each year [1]. Mild TBIs are non-penetrating impacts to the head that result in either a brief ( $<30 \mathrm{~min}$ ) loss of consciousness or an otherwise altered mental status $[1,2]$. While many who sustain a mild TBI fully recover within a few months, up to $60 \%$ continue to experience postconcussive symptoms for months or years after impact [1]. Symptoms often include generalized anxiety and post-traumatic stress disorder (PTSD)-like behaviors [1,3,4], which are mediated by brain regions such as the amygdala and the hippocampus [5]. These parts of the limbic system are particularly vulnerable to mechanical forces involved in mild TBI [1], and to cellular damage [5].

Many mild TBls occur in high-energy, stressful situations, such as combat, domestic abuse, traumatic accidents or sporting environments $[1,2,6]$. In such a state of arousal, glucocorticoids such as cortisol (corticosterone in rats) are elevated [7]. These steroids can bind to both glucocorticoid (GR) and mineralocorticoid (MR) receptors, found widely throughout the limbic system [8]. MRs have a higher affinity for cortisol/corticosterone than do glucocorticoid receptors (GRs) [8], thus MRs become saturated as glucocorticoid levels rise initially, with GRs becoming bound at higher concentrations. Activation of both receptors elicits changes in ion regulation and in neurotransmitter release and function [8].

There is some indirect evidence to suggest that glucocorticoids play a role in psychological symptoms induced by mild TBI. For example, GR antagonism has been shown to prevent neuron loss in the hippocampus after TBI in rats [9], a region known to be damaged by mild TBI and associated with resulting psychological symptoms [5]. Psychological stress at the time of injury may be partly indicative of the extent of postmild TBI impairment [6]. The GR antagonist mifepristone was also shown to be significantly better than placebo at treating symptoms of PTSD in military veterans [10]. Antagonism of MRs prior to injury has been shown in rats to reduce apoptotic damage in the hippocampus 24 hours after impact [9]. Furthermore, antagonism of either GRs or MRs has been shown to increase the amount of time spent in the open arms of an elevated plus maze in pre-stressed rats [8]. Notably, anxiety-like behavior in rats following a mild $\mathrm{TBI}$ concurrent with stress is more pronounced than after injury alone 
[7]. The ability of GR or MR antagonism to protect against neuronal injury caused by $\mathrm{TBI}$, combined with the anxiolytic effects of $\mathrm{GR}$ and MR blockade following stress, suggests that early targeting of one or both types of receptors may potentially abate anxiety resulting from stress-associated mild TBI.

Therefore, this experiment aimed to determine whether MRs or GRs play a differential role in post-mild TBI anxiety behaviors, via blockade of each receptor type. This was tested in a relevant rodent model of psychosocial stress with mild TBI, which reliably increases plasma corticosterone at the time of injury and elicits heightened anxiety-like behavior to a greater extent than either stress or mild TBI alone [7]. We show for the first time that $\mathrm{GR}$ antagonism is effective in reducing generalized anxiety caused by mild TBI incurred during stressful events.

These experiments were approved by the Institutional Animal Care and Use Committee of the University of South Dakota and all efforts were made to minimize the number of animals used and potential suffering. Forty-nine adult (8-10 weeks old) male Sprague-Dawley rats were housed on a reverse light cycle (12L:12D, lights off $10 \mathrm{AM}$ ) with food/water available ad libitum. All behavioral testing was performed under red lighting and commenced one hour after the dark cycle began.

Rats were acclimated to a solitary cage and the testing room (in preparation for social defeat) for $40 \mathrm{~min} /$ day for three days prior to the treatment day [7]. On the treatment day, rats received a single subcutaneous (sc.) injection of either vehicle (propylene glycol, $1 \mathrm{ml} / \mathrm{kg}$ ), the MR antagonist spironolactone (50 mg/kg [11]), or the GR antagonist mifepristone (20 mg/kg [11]), all purchased from Sigma-Aldrich (St. Louis, MO, United States), 40 min before exposure to either control (no stress/brain injury) or stress + mild TBI treatment ( $n=7-10$ per group). In a follow up study to determine whether any of the positive effects of mifepristone treatment in the stress/injury group were due to GR antagonism of the stress experience regardless of head injury, rats were treated with either mifepristone $(20 \mathrm{mg} / \mathrm{kg})$ or vehicle $30 \mathrm{~min}$ prior to social defeat and sham surgery ( $n=6-7$ per group). Drug concentrations were chosen based on efficacy in reducing anxiety-like behaviors in pre-stressed rats [11].

Forty minutes following vehicle or GR or MR antagonism, stress/injury or stress/sham rats underwent a single episode of social defeat by a larger, aggressive 
male rat ( $>100 \mathrm{~g}$ larger than subject), which reliably elevates plasma corticosterone [7] to create the desired conditions for mild TBI incurred under a stressed state. Control rats were placed in an empty cage similar to acclimation days (see Davies et al. 2015 [7] for protocol details).

Details of the surgical procedure to induce mTBI are given in Meyer et al. (2012) [5]. Details regarding the social defeat model coupled with the weight drop mild TBI are outlined in Davies et al. (2015) [7]. Briefly, at the conclusion of the defeat trial, rats were anesthetized with isoflurane (3-4\% in $\left.3.0 \mathrm{~L} / \mathrm{min} \mathrm{O}_{2}\right)$. The skin on the top of the head was shaved, disinfected, and a 1-inch long incision was made to expose the skull. A calibrated weight-drop device then delivered a 5,477 Newtons $/ \mathrm{m}^{2}$ impact to the exposed skull of the rat immediately behind bregma using a $175 \mathrm{~g}$ weight dropped from $42 \mathrm{~cm}$, with the force distributed across a $10 \mathrm{~mm}$ diameter area via a vertical transducer rod positioned just posterior to bregma and centered over the intraparietal suture. Induction of a mild traumatic brain injury was confirmed via delayed righting-reflex time postsurgery and by the absence of more severe damage (skull fracture, gross brain or vascular damage) at the conclusion of behavioral testing [5]. Control (sham) animals underwent surgery without receiving an impact.

Eight to nine days post-surgery, rats were evaluated for generalized anxiety using an elevated-plus maze (EPM) as described previously [5]. Rats were placed in the center of the maze facing the closed arms and allowed to explore freely for 5 minutes. Time spent in the open arms and total distance moved in the maze were measured using Ethovision XT V5.1 (Noldus Information Technology, Leesburg, VA USA).

A t-test compared righting reflex times and duration of time spent under anesthesia between control and defeat/mild TBI animals that had received vehicle. Two-way ANOVA (treatment $x$ drug) were used to analyze time spent in the open arms of the EPM, as well as distance traveled in the maze, with a priori comparisons made by one-way ANOVA or Student-Newman-Keuls (SNK) tests for multiple pairwise comparisons. One way ANOVA with SNK tests were used to compare the intensity of the social defeat event across the three drug treatment groups. For animals that experienced defeat without concussive injury, a t-test compared those treated with 
vehicle versus those that received mifepristone. The significance level was set at $\mathrm{P}<$ 0.05 throughout, and all analyses were performed using SigmaStat v11.0.

Vehicle-treated rats with mild TBI had a delayed righting reflex compared to vehicle-treated rats that underwent sham surgery $\left(t_{16}=2.396, P=0.029 ;\right.$ Fig. $\left.1 A\right)$, demonstrating presence of mild TBI [5]. With regard to EPM testing, there was a significant negative effect of treatment on time spent in open arms $\left(F_{2,41}=4.930 ; P=\right.$ 0.032). Furthermore, a priori pair-wise comparisons revealed that vehicle-treated rats subjected to defeat/mild TBI spent less time in open arms compared to vehicle-treated controls (SNK P $=0.028$ ), an effect that was not apparent in the mifepristone (SNK P = 0.855 ) or spironolactone (SNK $P=0.144$ ) groups (Fig. 1B). Subsequent one-way ANOVA to examine drug effects within injury group revealed no effect of drug in the control group $\left(F_{2,20}=0.004 ; P=0.996\right)$, but a drug effect in the defeat/mild TBI group $\left(F_{2,22}=3.772 ; P=0.039\right)$ (Fig. $\left.1 \mathrm{~B}\right)$. Rats treated with mifepristone prior to defeat $/ \mathrm{mild}$ TBI showed greater time in the open arms as compared to vehicle-treated rats (SNK $P$ $=0.031$; Fig. 1B). However, time spent in open arms did not differ between mifepristone and spironolactone injury groups nor between vehicle and spironolactone injury groups (SNK P > 0.05; Fig. 1B). These effects could not be accounted for by differences in time spent under isoflurane (control, mean+/- SEM = $18.3 \mathrm{~min}+/$ - 0.59; defeat $/ \mathrm{mild} \mathrm{TBI}$, mean+/- SEM $\left.=19.2 \mathrm{~min}+/-0.44, \mathrm{t}_{47}=1.170, \mathrm{P}=0.248\right)$. Similarly, intensity of the social defeat episode did not differ among rats that received either vehicle, mifepristone, or spironolactone $\left(n=26, F_{1,20}=0.256, P=0.777\right.$, data not shown), nor was there any difference in distance moved across all groups (drug, $F_{2,43}=1.034 ; P=0.364$, injury $F_{1,43}=2.336 ; P=0.134$, drug $x$ treatment $F_{2,43}=0.360 ; P=0.700 ;$ Fig. $\left.1 \mathrm{C}\right)$. In a followup study to address the possibility that it is not the effects of social defeat experience alone that are affected by the GR antagonist, animals that received social defeat, but not mild TBI exhibited similar times in open arms $(t=0.761, P>0.05)$ when treated with vehicle $(n=7$, mean $=16.6 \mathrm{sec} \pm$ SEM 10.1) and mifepristone $(n=6$, mean $=7.6 \mathrm{sec} \pm$ SEM 4.8).

Our results indicate that the GR antagonist mifepristone was able to restore anxiety-like behavior to control levels in animals that had experienced social defeat concurrent with mild TBI. While MR antagonism prior to injury partially appeared to 
reverse anxiety-like behavior in these animals, the effect was not different from those treated with vehicle. These results suggest that GRs, more so than MRs, mediate anxiety behaviors that arise following stress with mild TBI. It was also found that GR antagonism does not alter the effects of social defeat alone on anxiety behavior-only the combined anxiogenic effect of social defeat and mild TBI was mitigated by mifepristone. This indicates that blockade of GRs showed a positive therapeutic outcome for concussive injury, acting to subvert the development of anxiety resulting from mild TBI in stressful conditions.

In the past, glucocorticoids have been used as a post-injury therapy for head traumas [12]. Those recommendations have since been updated to advise against this approach [13], a change supported by our findings. Glucocorticoids have antiinflammatory properties, but their actions as stress signals in the brain can have undesired side effects. Pairing a stressor (social defeat) that increases corticosterone levels with mild TBI (as in the current study) produces significantly worse psychological symptoms than does mild TBI alone [7]. Furthermore, both GRs and MRs are known to promote cell death following TBI, and mifepristone and spironolactone can decrease activation of these apoptotic pathways [9]. Combined with the current results, this suggests that stress and the molecular actions of its steroid messengers are harmful to the brain during physical trauma, and that beneficial effects are more likely achieved with GR blockade rather than activation following TBI. Possible mechanisms for positive outcomes of GR blockade include interruption of retrieval of traumatic memories that cause anxiety [14], or the prevention of neuronal death in key limbic brain regions known to be susceptible to mild TBI, such as the hippocampus [5,9]. Opportunities are ripe for further studies of how glucocorticoid antagonism might enhance limbic neuron survival and modulate emotional memory following concussion.

Spironolactone delivery prior to injury partially reduced anxiety-like behavior following stress and mild TBI. A dose-response study incorporating each antagonist may reveal that higher doses of spironolactone are in fact as effective as mifepristone was in this study. Further work to investigate successful dosing schedules and time of injection is merited. 
Here, we delivered mifepristone and spironolactone prior to stress and mild TBI exposure, allowing identification of which type of glucocorticoid receptor was most responsible for affecting subsequent anxiety. However, while this approach elucidates the mechanisms of stress in concussion at the time of injury, it does not provide an optimal treatment paradigm. It is not practical to give mifepristone prophylactically in all situations where head trauma is a risk, but our findings do suggest that targeting glucocorticoid receptors could be a viable treatment for preventing anxiety specifically caused by mild TBI. Future studies will explore whether mifepristone is as effective when given post-injury. Anxiety is one of many behavioral changes precipitated by mild TBI, so further investigation of how mifepristone can mitigate other symptoms such as PTSD-like behaviors or even cognitive impairments is certainly warranted. Treatments that reduce glucocorticoid receptor signaling hold promise for victims of mild TBI, and represent a potential path for preventing some of this injury's most debilitating psychological symptoms.

Overall, the ability of mifepristone to prevent anxiety-like behavior in rats when delivered prior to mild TBI with concurrent stress indicates that GRs are responsible in part for some of the emotional symptoms associated with mild TBI. Pharmacotherapies for psychological impairments following this type of injury may be most effective if targeted to GRs.

\section{Acknowledgements:}

The authors would like to thank Nate J. Vinzant for his contributions to the experiment. Funding was provided by the United States Department of Defense, Grant W81XWH-10-1-0578 to Dr. Gina L. Forster, and Laura C. Fox was supported by the University of South Dakota Medical Student Summer Science Research Program.

\section{References:}

[1] Moore, E. L., Terryberry-Spohr, L., \& Hope, D. A. (2006). Mild traumatic brain injury and anxiety sequelae: a review of the literature. Brain Injury 20(2): 117-132.

[2] Kennedy, J. E., Jaffe, M. S., Leskin, G. A., Stokes, J. W., Leal, F. O., \& Fitzpatrick, P. J. (2007). Posttraumatic stress disorder and posttraumatic stress disorder-like 
symptoms and mild traumatic brain injury. The Journal of Rehabilitation Research and Development, 44(7), 895-920.

[3] Gaylord, K. M., Cooper, D. B., Mercado, J. M., Kennedy, J. E., Yoder, L. H., \& Holcomb, J. B. (2008). Incidence of posttraumatic stress disorder and mild traumatic brain injury in burned service members: preliminary report. Journal of Trauma, 64(2 Suppl), S200-205; discussion S205-206.

[4] Hoge, C. W., McGurk, D., Thomas, J. L., Cox, A. L., Engel, C. C., \& Castro, C. A. (2008). Mild Traumatic Brain Injury in U.S. Soldiers Returning from Iraq. New England Journal of Medicine, 358(5), 453-463.

[5] Meyer, D. L., Davies, D. R., Barr, J. L., Manzerra, P. \& Forster, G. L. (2012). Mild traumatic brain injury in the rat alters neuronal number in the limbic system and increases conditioned fear and anxiety-like behaviors. Experimental Neurology 235(2): 574-587.

[6] Bay, E. H., \& Liberzon, I. (2009). Early Stress Response: A Vulnerability Framework for Functional Impairment Following Mild Traumatic Brain Injury. Research and Theory for Nursing Practice, 23(1), 42-61.

[7] Davies, D. R., Olson, D. M., Meyer, D. L., Scholl, J. L., Watt, M. J., Manzerra, P., Renner, K. J. \& Forster, G. L. (2016). Mild Traumatic Brain Injury with Social Defeat Stress Alters Anxiety, Contextual Fear Extinction, and Limbic Monoamines in Adult Rats. Frontiers in Behavioral Neuroscience (10), 71. doi: 10.3389/fnbeh.2016.00071

[8] Korte, S. M., De Boer, S.F., De Kloet, J. E. R., \& Bohus, B. (1995). Anxiolytic-like effects of selective mineralocorticoid and glucocorticoid antagonists on fear-enhanced behavior in the elevated plus-maze. Psychoneuroendocrinology 20(4): 385-394.

[9] McCullers, D. L., Sullivan, P. G., Scheff, S. W., \& Herman, J. P. (2002). Mifepristone protects CA1 hippocampal neurons following traumatic brain injury in rat. Neuroscience, 109(2), 219-230.

[10] Golier, J. A., Caramanica, K., Demaria, R., \& Yehuda, R. (2012). A pilot study of mifepristone in combat-related PTSD. Depression Research and Treatment, 2012, 393251.

[11] Adamec, R., Muir, C., Grimes, M., \& Pearcey, K. (2007). Involvement of noradrenergic and corticoid receptors in the consolidation of the lasting anxiogenic effects of predator stress. Behavioural Brain Research, 179(2), 192-207.

[12] Ghajar, J., Hariri, R. J., Narayan, R. K., lacono, L. A., Firlik, K., \& Patterson, R. H. (1995). Survey of critical care management of comatose, head-injured patients in the United States. Critical Care Medicine, 23(3), 560-567. 
[13] Huizenga, J. E., Zink, B. J., Maio, R. F., \& Hill, E. M. (2002). Guidelines for the management of severe head injury: are emergency physicians following them? Academic Emergency Medicine, 9(8), 806-812.

[14] Tronel, S., \& Alberini, C. M. (2007). Persistent disruption of a traumatic memory by postretrieval inactivation of glucocorticoid receptors in the amygdala. Biological Psychiatry, 62(1), 33-39. 


\section{Figure Legend:}

\section{Figure 1 - Behavioral effects of mild traumatic brain Injury concurrent with}

psychosocial stress. A: Righting reflex time after anesthesia is delayed in vehicletreated rats with mild TBI ( ${ }^{*} \mathrm{P}<0.05 ; \mathrm{n}=7-8$ per group). $\mathrm{B}$ : Time spent in the open arms of the elevated plus maze was reduced following exposure to defeat/mild TBI, which was prevented by mifepristone and partially prevented by spironolactone treatment ( ${ }^{*}$ different from vehicle-treated controls; \# different from vehicle-treated SD/mild TBI group; $\mathrm{P}<0.05 ; \mathrm{n}=7-10$ per group). C: Mild TBI or drug treatment did not alter distance moved in the elevated plus maze. $\mathrm{SD}=$ social defeat, $\mathrm{mTBI}=$ mild traumatic brain injury. 

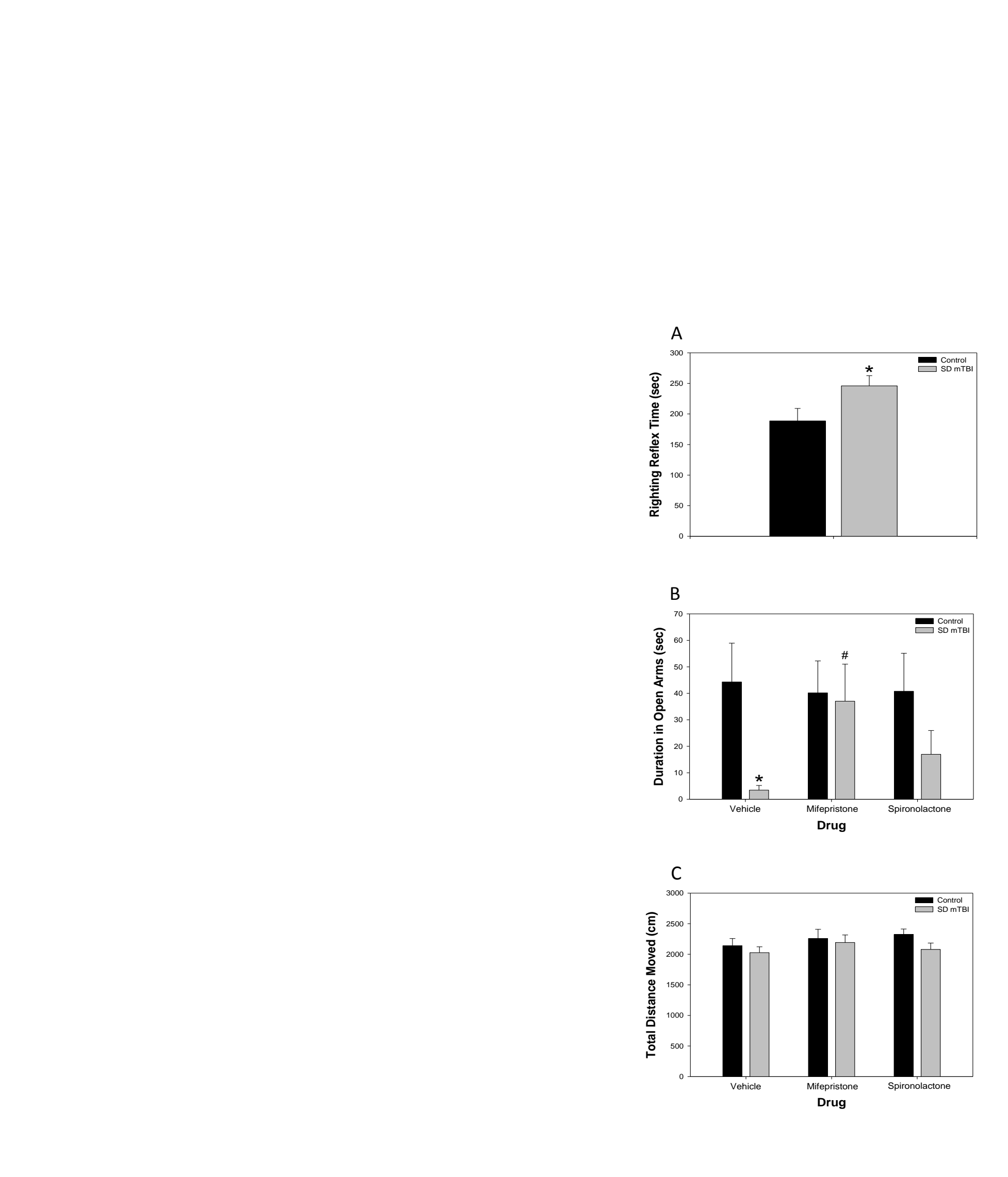

. 